\title{
Canadian Collaborative Project on Genetic Susceptibility to MS, Phase 2: Rationale and Method
}

\author{
A.D. Sadovnick, N.J. Risch, G.C. Ebers and the Canadian \\ Collaborative Study Group *
}

\begin{abstract}
Background: Results from the Canadian Collaborative Project on Genetic Susceptibility to Multiple Sclerosis (MS)-Phase 1 (CCPGSMS-Phase 1) together with other family data published since 1982 have led to the following conclusions about the etiology of MS: (i) genetic and non-genetic (environmental) factors are involved in the etiology of MS on a population basis; (ii) the familial aggregation of MS is genetic; (iii) maternal factors do not influence the risk for siblings to develop MS; and (iv) MS appears to be oligogenic. The present paper describes the rationale and methodology for the CCPGSMS-Phase 2. Method: The CCPGSMS-Phase 2 is a nation-wide collaborative effort involving all the 15 Canadian MS clinics. A series of structured questionnaires is administered to MS index cases, spouse controls and mothers of index cases and spouse controls (if available) by trained interviewers. Blood samples are taken for molecular genetic studies. This national effort is coordinated by the MS Clinics in Vancouver and London. Results: The CCPGSMS-Phase 2 is in progress so specific results are not available. The study is designed to (i) increase the database for genetic epidemiological/molecular genetic research and (ii) gather population-based data to further our understanding of the non-genetic factors in the etiology of MS. Conclusions: It is anticipated that the results from this study will impact on the eventual prevention, cure and treatment of MS.
\end{abstract}

RÉSUMÉ: Projet canadien multicentrique sur la susceptibilité génétique à la SEP, phase 2: Justification et Méthode. Introduction: Les conclusions suivantes sur l'étiologie de la sclérose en plaques (SEP) découlent des résultats du projet collaboratif canadien sur la susceptibilité génétique à la SEP phase 1 (CCPGSMS - phase 1) ainsi que d'autres données familiales publiées depuis 1982: (1) des facteurs génétiques et non génétiques (environnementaux) sont impliqués dans l'étiologie de la SEP dans la population; (2) l'agrégation familiale des cas de SEP est génétique; (3) les facteurs maternels n'influencent pas le risque de développer la SEP dans la fratrie; et (4) la SEP semble oligogénique. Nous décrivons la justification et la méthodologie de la phase 2 du CCPGSMS. Méthode: il s'agit d'une étude collaborative pancanadienne impliquant les 15 cliniques canadiennes de SEP. Une série de questionnaires structurés est administrée aux cas index de SEP, aux conjoints servant de contrôles et aux mères des cas index et à leurs conjoints contrôles (si disponibles) par des interviewers entrânés. Des échantillons sanguins sont recueillis pour des études génétiques. Cette étude nationale est coordonnée par les cliniques de SEP de Vancouver et de London. Résultats: La phase 2 du CCPGSMS est en cours et les résultats ne sont donc pas disponibles. L'étude a été conçue pour (1) augmenter la base de données pour la recherche en épidémiologie génétique/génétique moléculaire et (2) pour recueillir des données de population afin de parfaire notre compréhension des facteurs non génétiques dans l'étiologie de la SEP. Conclusions: Nous nous attendons à ce que les résultats de cette étude influencent la prévention éventuelle, la prise en charge et le traitement de la SEP.

Can. J. Neurol. Sci. 1998; 25: 216-221

The Canadian Collaborative Project on Genetic Susceptibility to Multiple Sclerosis-Phase 1 (CCPGSMS-Phase 1) is the largest population based MS study to date. The CCPGSMSPhase 1 was designed to execute studies which were comprehensive and definitive. The results (i) validated the potential of a Canadian nation-wide collaborative effort and (ii) answered fundamental questions about the relative roles of genetic (inherited) and non-genetic (environmental) factors in the etiology of multiple sclerosis (MS) - see ${ }^{1-3}$. Results of the CCPGSMS-Phase 1, together with other family data published since 1982 (e.g., studies of twins ${ }^{4-6}$ and familial risk data ${ }^{7-9}$ ) have led to the following conclusions about the etiology of MS: (i) genetic and non-genetic (environmental) factors are involved in the etiology of MS on a population basis; (ii) the familial aggregation of MS is genetic; (iii) maternal factors do not influence the risk for sibs to develop MS; (iv) MS appears to be oligogenic - more than one locus. It remains to be seen whether a major locus, with the possible exception of HLA, exists. ${ }^{3,10,11}$
Concurrent with the CCPGSMS-Phase 1, three groups simultaneously published initial data from full genome screens in MS, ${ }^{3,10,11}$ but much work remains to be done.

We are now in the fortunate position to continue the Canadian Collaborative Project on Genetic Susceptibility to MS into Phase 2 (CCPGSMS-Phase 2) which is designed to (i) increase

From the Department of Medical Genetics, University of British Columbia, Vancouver (A.D.S.); the Department of Genetics, Stanford University School of Medicine, California, U.S.A. (N.J.R.); Department of Clinical Neurological Sciences, University of Western Ontario, London (G.C.E.).

RECEIVED DECEMBER 8, 1997. ACCEPTED IN FINAL FORM MARCH 18, 1998.

* Contributors: the Canadian Colloborative Study Group - L. Metz (Calgary AB); S Warren (Edmonton AB); W. Hader (Saskatoon SK); C. Power (Winnipeg MB); P O'Connor (Toronto ON); R. Paulseth (Hamilton ON); D. Brunet (Kingston ON); R. Nelson (Ottawa ON); G. Francis (Montreal QC); P. Duquette (Montreal QC); J.P. Bouchard (Quebec City QC); T.J. Murray (Halifax NS); W. Pryse-Phillips (St. John's, NF); S.A. Hashimoto, J.J-F. Oger, D.W. Paty (Vancouver BC); G. Rice (London ON). Reprint requests to: A. Dessa Sadovnick, GF401 Koerner Pavilion, VHHSSC-UBC 2211 Wesbrook Mall, Vancouver, British Columbia, Canada V6T 2B5 
the database for genetic epidemiological/molecular genetic research and (ii) gather population-based data to further our understanding of the non-genetic (environmental) factors in the etiology of MS.

Given the vast literature on the role of genetics in MS, it is unnecessary to present in this paper any rationale for continuing genetic studies as part of the CCPGSMS-Phase 2.

The findings from the CCPGSMS-Phase 1 clearly show that the familial aggregation of MS (i.e., increased MS concordance among biological relatives) is not due to shared "family environment", but rather to genetic sharing among these individuals. ${ }^{1,2}$ It is thus now imperative to refocus research on non-genetic (environmental) factors away from the "within family" historical approach of investigating non-genetic transmission factors as has been studied by looking at, for example, birth order position to environmental factors which can act at the population level.

From the CCPGSMS-Phase 1 and other research, we have come to this conclusion because no risk can be attributed to: (i) commonality of uterine environment, e.g., dizygotic twin rate $=$ non-twin sib rate; $;^{4-6}$ maternal $1 / 2$ sib rate = paternal $1 / 2 \mathrm{sib}$ rates; ${ }^{2}$ (ii) early life environment, e.g.: no increased risk for "nonbiological" (adopted) sibs' or half-sibs raised together compared with half-sibs raised apart; ${ }^{2}$ or (iii) later life environment. ${ }^{12}$ Indeed, the "adoptee" data ${ }^{1}$ provide no evidence of risk transfer (i) from non-biological parent to child, (ii) from nonbiological child to parent or (iii) among non-biological sibs.

Taken together, the implications of the data we have briefly discussed here and the results of the CCPGSMS-Phase 1 imply a major environmental factor(s) which operates broadly at the population level conferring differing risks on susceptible individuals depending on where they live. We cannot formally exclude infrequent chance events as either a precipitant(s) of the disease or any stochastic model in which genes are necessary, but require an accumulation or concentration of environmental (chance) events to exceed a disease triggering threshold. Such "triggering" would require the coincidence of several factors (e.g., age, hormonal status, background immunological stimulation, etc.) having an element of chance or randomness including epigenetic factors such as somatic mutation. Approaches to address these possibilities have, to date, been largely sterile, with the exception of the well-known approximate 1.5-2:1 female/male ratio in $M^{13}$ and the clear evidence that viral infections may precipitate MS relapses. ${ }^{14}$ It is not clear if the prevalence of viral infections accounts, even in part, for regional differences in MS. Factors triggering onset and those triggering relapses are probably not the same and may, in fact, clearly differ from the hypothesized early life factors influencing subsequent susceptibility.

There are relatively few broad factors which would act at the level of the population to differentiate "at risk" groups. Indeed, climate (or its indirect consequences), diet or these two together seem the most attractive possibilities. Dietary differences have indeed been extensively investigated without firm conclusions about any particular foodstuffs, toxins or deficiencies that predispose individuals to developing MS. On the other hand, differences in climate are easily recognized and enumerated. These show high correlations with MS susceptibility. However, approaches to date have largely stopped here. Although demonstrating an association with climate, latitude, sunshine, etc., they have been ecological in nature and lacking the power either to differentiate among these or to suggest the nature of any causal relationship. Our interpretation of the available data, including those on migrants, is that MS susceptibility is determined at an early age. Accordingly it may well be that the timing of putative environmental factors occurs early (and perhaps very early) in the life of a person who develops MS.

We believe that the database for the CCPGSMS-Phase 2 will allow the dissection of early life experience for MS patients with a view to identifying infrequent events which are either over-represented (i.e., predisposing) or under-represented (i.e., protective) among the MS population compared to a control population. The size of the CCPGSMS-Phase 2 database suggests that at the very least, a reasonable expectation can be that many factors long debated to have a causal role in MS could be definitively excluded. No previous study has had similar statistical power to detect an over- or under-representation of infrequent, time-specific events.

Identifying environmental risk factors may well lead to a rational method of preventing MS since data from twins suggest that a subtle balance exists between susceptibility and manifest expression. We believe that should these studies prove to be negative, the ground will have been covered in a sufficiently definitive manner to lead to meaningful conclusions.

The epidemiological/ecological approach outlined is the only feasible one at the moment. It is recognized that this can, at the best, provide indirect information bearing on these questions. However, it is expected that this information will lead to more specific hypotheses.

\section{Methodology - CCPGSMS-Phase 2}

The CCPGSMS-Phase 2 consists of 3 study groups:

(i) CCPGSMS-Phase 1 index cases ("Phase I index cases") refers to the approximately 20,000 active Canadian MS clinic patients who were screened as part of the CCPGSMS-Phase 1;

(ii) CCPGSMS-Phase 2 index cases ("Phase 2 index cases") refers to all persons who first presented to a Canadian MS Clinic after June 30, 1993, i.e., the cut-off date for the CCPGSMSPhase 1 , and who were given a diagnosis of MS according to recognized criteria ${ }^{15}$;

(iii) CCPGSMS-Phase 2 controls ("Phase 2 controls") refers to spouses or equivalent (i.e., common law; significant other regardless of gender, etc.) of index cases.

The CCPGSMS-Phase 1 was based on the caseload for 14 MS clinics. The CCPGSMS-Phase 2 is now being carried out at 15 MS clinics since the Quebec City MS Clinic was only officially established after completion of the CCPGSMS-Phase 1. All cases from that clinic are thus, by definition, Phase 2 index cases.

Each MS clinic participating in the CCPGSMS-Phase 2 has received ethical approval from the relevant institution (i.e., hospital, university). In addition, the University of British Columbia has been given ethical approval for the entire project. All informed consent forms for data collection and blood sampling were approved by the appropriate committees.

As was successfully done for the CCPGSMS-Phase 1, all interviews for the CCPGSMS-Phase 2 are administered by telephone by the site research nurse (or equivalent) - hereafter referred to as "research nurse". An " 800 " number for the Vancouver coordinating centre provides ongoing support for all research nurses who have been trained by personnel from Vancouver. 


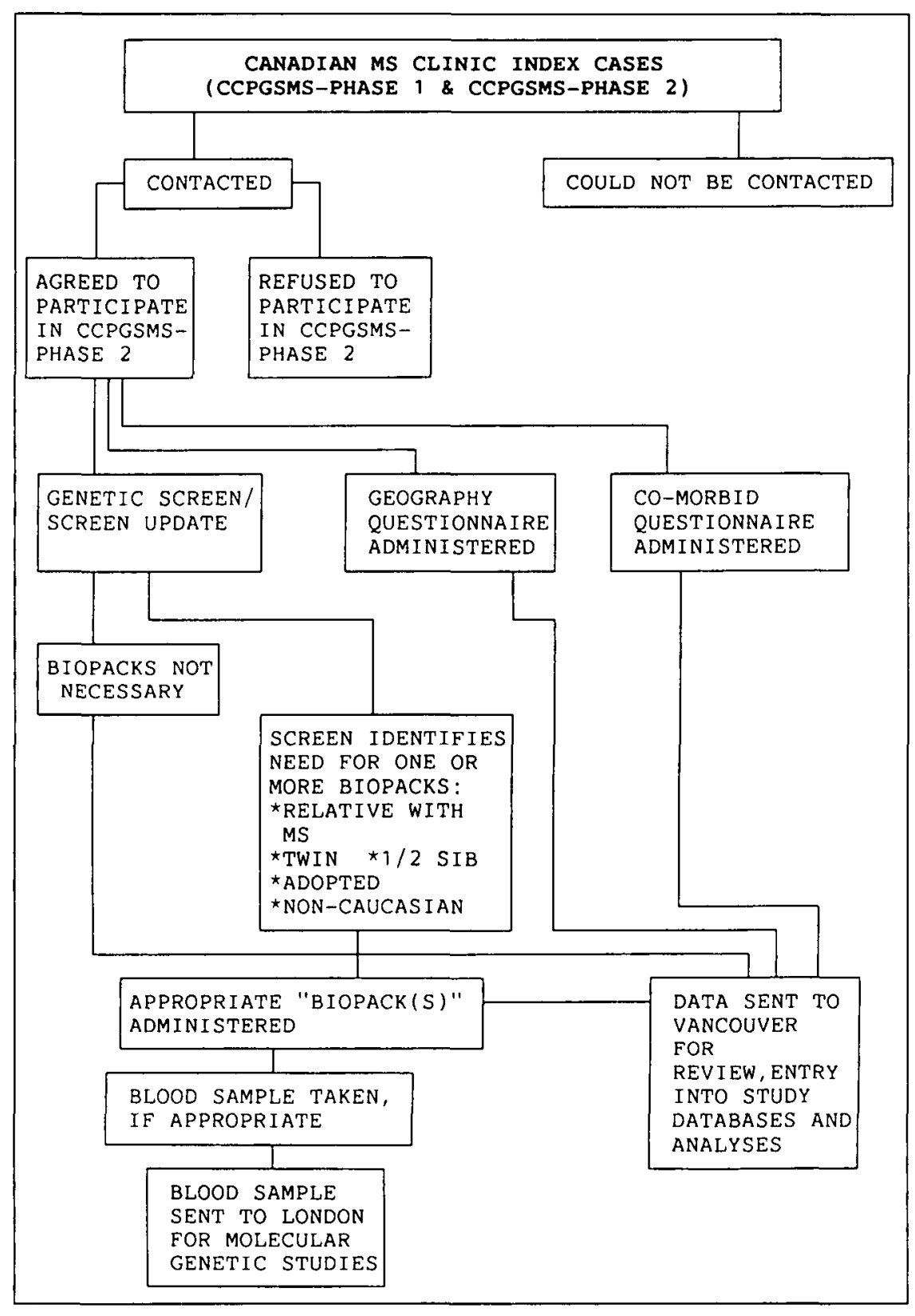

Figure 1: FLOW CHART: Phase 1 and Phase 2 Index Cases: Screen Summary (Update Questionnaires, Geography Questionnaire, Co-Morbid Questionnaire.

Interviews are conducted using standardized questionnaires. Each patient is interviewed by the research nurse from the MS clinic he/she regularly attends. For example, an individual attending the Kingston, Ontario MS Clinic is interviewed by personnel from that particular clinic. As shown in the CCPGSMS-Phase 1 , this results in an exceptionally high rate of cooperation (exceeding 95\%).

\section{Phase 1 Index Cases}

As shown in Figure 1, each "Phase 1 index case" is recontacted. If he/she agrees to the interview, the questionnaires briefly summarized below (see Table 1) are administered.

Genetic Screen Update Questionnaire (see Figure 1): This questionnaire is designed to determine whether there has been any change in the family history since the initial interview done as part of the CCPGSMS-Phase 1. For example, this questionnaire could identify a new case of MS in the family, a death by suicide, etc. If a change(s) is noted, appropriate biopacks are administered to fully document and record this information. "Biopacks" refer to structured interviews which document family structure and family history. For example, the "sib biopack" documents the following information about full sibs (same mother and father) of the index case: (i) name; (ii) date of birth; (iii) date of death and cause of death (if applicable); (iv) gender; (v) MS present or absent; and (vi) any significant medical problems other than MS.

Geography Questionnaire \& Co-Morbid Questionnaire (see Figure 1): Each Phase 1 index case is administered a geography questionnaire which records his/her place of birth and documents subsequent significant moves. A significant move, for the purpose of this study, is defined as one which (i) lasted at least 12 months and (ii) was at least 500 miles from the previous place of residence. Each Phase 1 index case is also given the "co-morbid questionnaire" which asks about the presence or absence of other diseases, including autoimmune diseases such as systemic lupus erythematosus, rheumatoid arthritis and juvenile diabetes.

Early Life Questionnaire (see Figure 2): Each CCPGSMS-Phase 1 index case is asked if he/she has a mother who can provide information on early life. The term "mother" can be somewhat arbitrary and we recognized that family relationships can vary. Nevertheless, for the purpose of the CCPGSMS-Phase 2, it is necessary to clearly define the term and thus we specifically refer to the biological mother of the index case or spouse control. The Early Life Questionnaire is only administered if a reliable maternal informant (e.g., living, cognitively unimpaired, raised the child) is available and is willing to participate.

The Early Life questionnaire focuses on gestation and delivery, the perinatal period, the preschool period and the period encompassing kindergarten and primary school. The "gestation and delivery" questions include items about prematurity, birthweight and length of hospitalization. The questions on the perinatal period include items about birth defects, jaundice, blood group incompatibility and allergies. The questions about the preschool and kindergarten/primary school periods focus on infections, allergies, vaccinations and overall health issues. Questions are also asked about puberty and major trauma.

Questionnaires Administered to an Index Case's Spouse or Equivalent (see Figure 3): Each Phase 1 index case is asked whether or not he/she has a spouse ("spouse control") who is willing to be a control for the study. If the answer is "yes", the spouse is contacted and administered the appropriate questionnaires - see below and 


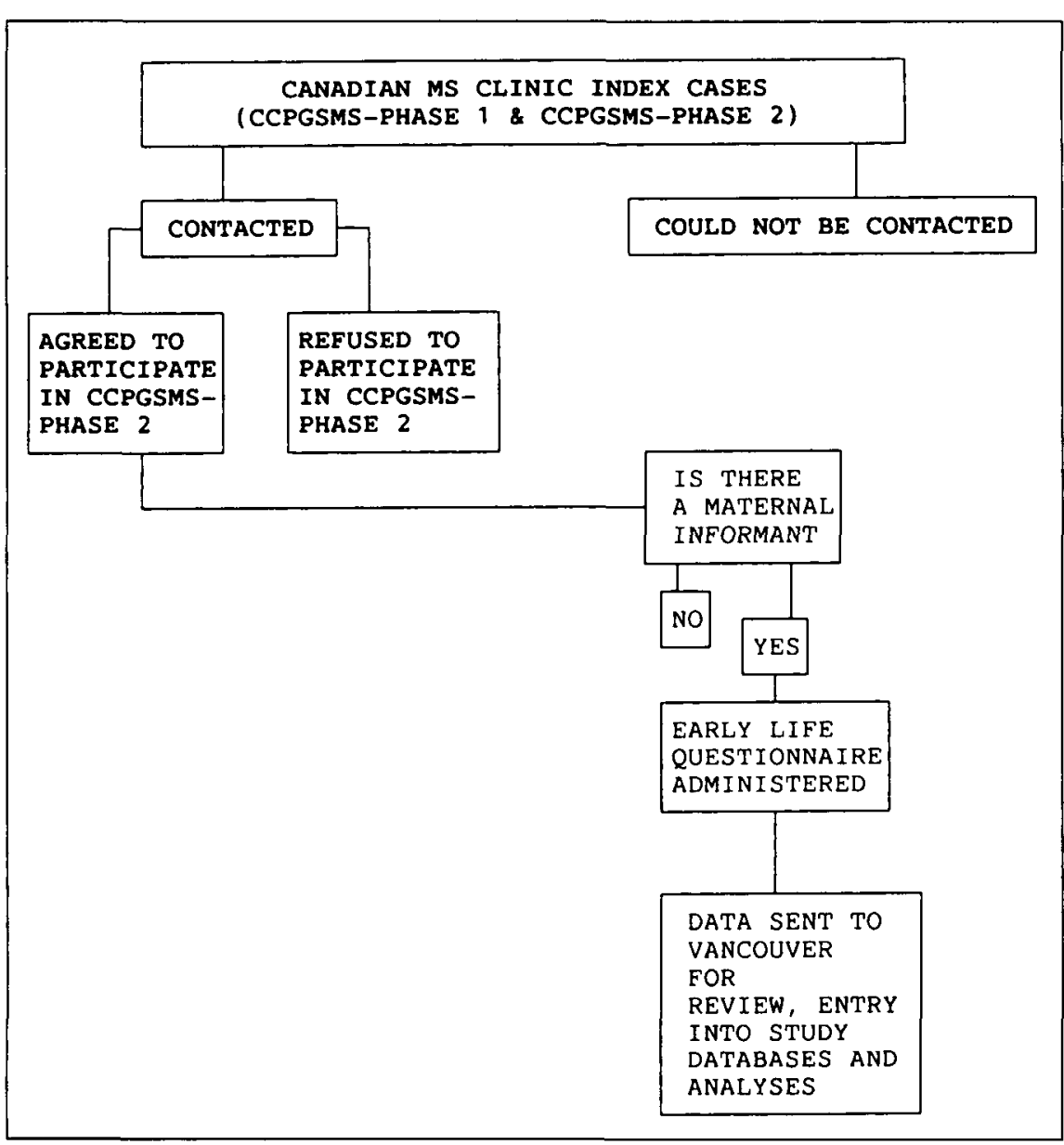

Figure 2: FLOW CHART: Phase 1 and Phase 2 Index Cases: Early Life Questionnaire.

Table 1: Summary of Questionnaires/Data Forms.

\begin{tabular}{|c|c|c|c|c|c|}
\hline & $\begin{array}{l}\text { Index } \\
\text { Case }\end{array}$ & $\begin{array}{l}\text { Spouse } \\
\text { Control } \\
\text { Blood } \\
\text { Sample } \\
\text { Drawn }\end{array}$ & $\begin{array}{l}\text { Affected } \\
\text { Relative, } \\
\text { Blood } \\
\text { Sample } \\
\text { Drawn }\end{array}$ & $\begin{array}{l}\text { Unaffected } \\
\text { Relative, } \\
\text { Blood } \\
\text { Sample } \\
\text { Not Drawn" }\end{array}$ & $\begin{array}{l}\text { Affected } \\
\text { Relative, }\end{array}$ \\
\hline $\begin{array}{l}\text { Genetic Screen/ } \\
\text { Update }\end{array}$ & $Y^{\mathbf{b}}$ & $Y$ & $\mathrm{~N}^{\mathrm{c}}$ & $\mathrm{NN}$ & \\
\hline Geography & $\mathrm{Y}$ & $\mathrm{Y}$ & $\mathrm{N}$ & $\mathrm{N}$ & $\mathrm{N}$ \\
\hline Co-Morbid & $\mathrm{Y}$ & $\mathrm{Y}$ & $\mathrm{N}$ & $\mathrm{N}$ & $\mathrm{N}$ \\
\hline Clinical Summary & $\mathrm{Y}$ & $\mathrm{N}$ & $\mathrm{Y}$ & $\mathrm{N}$ & $\mathrm{Y} / \mathrm{N}^{\mathrm{d}}$ \\
\hline Biopack(s) & $\mathrm{Y} / \mathrm{N}^{\mathrm{e}}$ & $\mathrm{Y} / \mathrm{N}^{\mathrm{e}}$ & $\mathrm{N}$ & $\mathrm{N}$ & $\mathrm{N}$ \\
\hline Early Life & $\mathrm{Y} / \mathrm{N}^{\mathrm{e}}$ & $\mathrm{Y} / \mathrm{N}^{\mathrm{e}}$ & $N$ & $\mathrm{~N}$ & $\mathrm{~N}$ \\
\hline $\begin{array}{l}\text { Confirmation of } \\
\text { MS Diagnosis } \\
\text { or Unaffected }\end{array}$ & & & & & \\
\hline Status & $Y$ & $Y$ & $\mathrm{Y}$ & $\mathrm{Y}$ & $\mathrm{Y}$ \\
\hline
\end{tabular}

"Blood sample was not drawn. Reasons for this include refusal, death of affected relative, family dynamics, etc.

b"Y" refers to YES;

"N" $N$ " refers to NO.

dSufficient data to complete the clinical summary may be unavailable, although the diagnosis of MS could be confirmed.

'These are completed if appropriate.
Figure 3. For the purpose of the CCPGSMSPhase 2, the term spouse refers to a legal spouse, same-sex partner or common-law partner.

\section{Phase 2 Index Cases}

As shown in Figure 1, each contacted CCPGSMS-Phase 2 index case who agrees to participate is given the following questionnaires (see Table 1): Genetic Screen Questionnaire (see Figure 1): This questionnaire is designed to determine whether the index case is of special interest for at least one of the following reasons: (i) the index case has/had a family member with MS; (ii) index case is adopted; (iii) the index case has/had an adopted/adoptive sib(s); (iv) the index case has/had an adopted child(ren); (v) the index case is/was a twin; (vi) the index case has/had a half sib(s); and/or (vii) the index case is not caucasian. As done in the CCPGSMS-Phase 1, family history information is documented through the administration of appropriate "biopacks".

Geography Questionnaire \& Co-Morbid Questionnaire (see Figure 1): Each Phase 2 index case is given the geography questionnaire and the co-morbid questionnaire, as described in the section on "CCPGSMS-Phase 1 Index Cases".

Early Life Questionnaire (see Figure 2): Each CCPGSMS-Phase 2 index case is asked whether or not he/she has a mother who can provide information on early life ("Early Life Questionnaire"), as described in the section on "Phase 1 Index Cases".

Questionnaires Administered to an Index Case's Spouse or Equivalent (see Figure 3): Each CCPGSMS-Phase 2 index case is asked whether or not he/she has a spouse or equivalent ("spouse control") who is willing to participate. If the answer is "yes", the spouse control is contacted and administered the appropriate questionnaires - see below and Figure 3.

\section{Phase 2 Spouse Controls}

As shown in Figure 3, each "spouse" who agrees to participate is given the following questionnaires (see Table 1): Genetic Screen Questionnaire: This questionnaire is designed to determine whether the spouse control is of special interest because of any of the following reasons: (i) the spouse has a family member with MS; (ii) the spouse is adopted; (iii) the spouse has/had an adopted/adoptive sib(s); (iv) the spouse has/had an adopted child(ren); (v) the spouse is/was a twin; (vi) the spouse has/had a half sib(s); and/or (vii) the spouse is not caucasian. If the spouse meets at least one of the above categories, the family history will be documented through the administration of appropriate "biopacks", as done for index cases. 


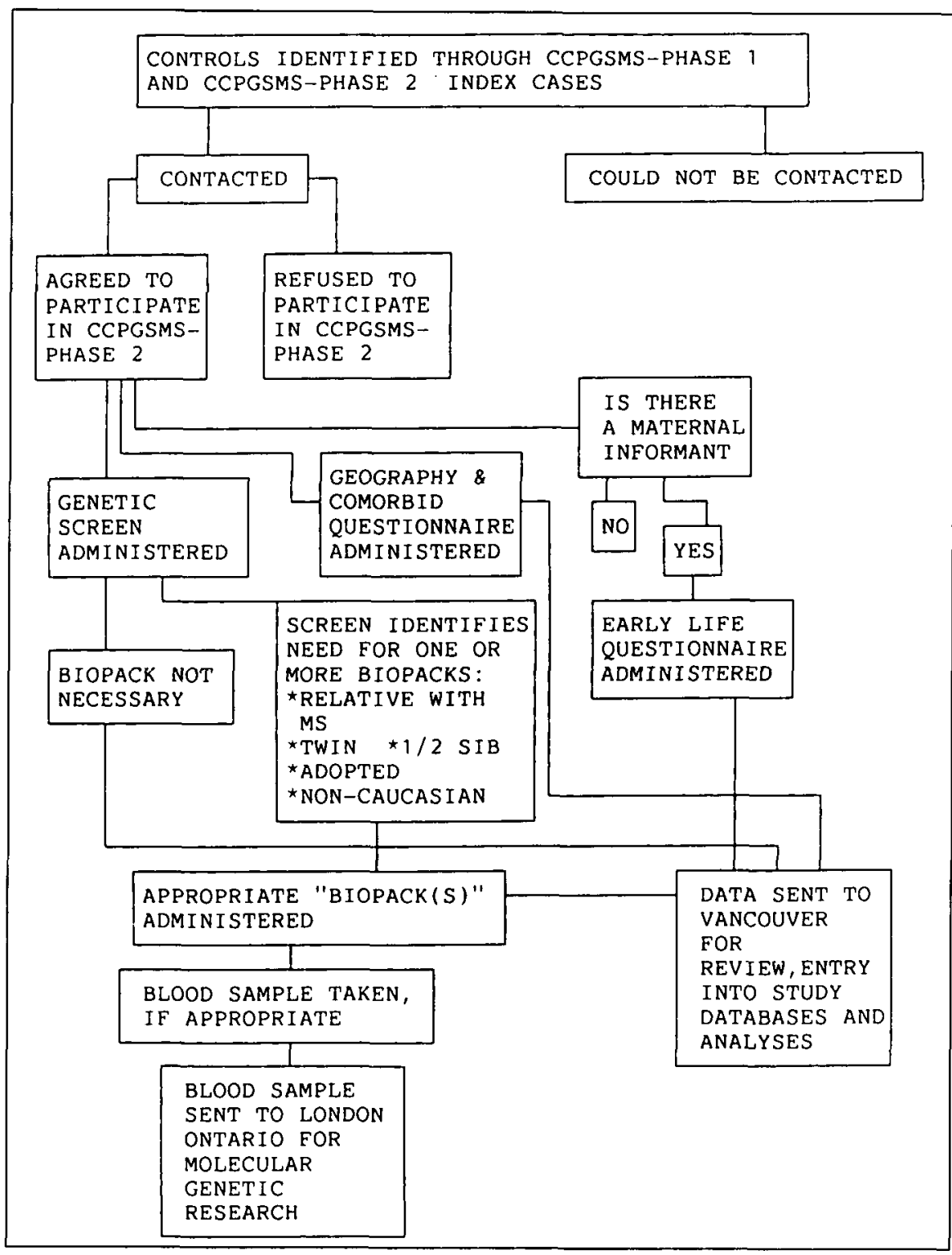

Figure 3: FLOW CHART: Spouse Controls.

elsewhere, i.e., clinical/autopsy records and/or assessment at a Canadian MS clinic.'4,7 For each affected relative for whom a blood sample was drawn, a clinical summary with respect to MS was obtained from both a review of medical records and discussion with the relative.

Unaffected Relative, Blood Sample Drawn: Confirmation of "unaffected" status with respect to clinical MS was done according to the protocol described elsewhere. ${ }^{1-4,7}$

Affected Relative, Blood Sample Not Drawn: Blood sample could not be drawn from affected family members for a variety of reasons including death of the affected relative, family dynamics, etc. Although the confirmation of MS diagnosis was done according to the protocol described elsewhere, i.e., clinical/autopsy records and/or assessment at a Canadian MS clinic, ${ }^{1-4,7}$ it was not always possible to obtain sufficient information to complete a clinical summary.

\section{CONCLUSION}

In conclusion, the CCPGSMS-Phase 2 is now in progress. As with the CCPGSMSPhase 1, great care is taken to confirm the "affected" or "unaffected" status with respect to MS for relatives of index cases. Data collection is underway at all the 15 Canadian MS clinics. It is anticipated that the results of this country-wide, population-based study will significantly add to our understanding about the etiology of MS.

\section{ACKNOWLEDGEMENTS}

This research was funded by the Multiple Sclerosis Society of Canada Scientific Research Foundation and the Multiple Sclerosis Society of Canada.

The following MS Clinic research nurses (or equivalent) were responsible for accurate data col-

Geography Questionnaire \& Co-Morbid Questionnaire:

Each spouse control is given the geography questionnaire and the co-morbid questionnaire, as described in the section on "CCPGSMS-Phase 1 Index Cases".

Early Life Questionnaire: Each spouse control is asked whether or not he/she has a mother who can provide information on early life ("Early Life Questionnaire"), as described in the section on "CCPGSMS-Phase 1 Index Cases".

\section{Confirmation of "Affected"/"Unaffected" MS Status}

An essential component of the CCPGSMS (Phases 1 and 2) is confirmation of the diagnosis of MS, a clinical summary which includes age of MS onset, MS course (e.g., relapsing/remitting, relapsing/progressive, primary progressive), disability (e.g., EDSS $\left.^{16}\right)$ and first symptom(s) and confirmation that an unaffected family member is indeed unaffected (see Table 1).

Affected Relative, Blood Sample Drawn: Confirmation of MS diagnosis was done according to the protocol described lection*: D. Bucciarelli (London); L. Mashal, E. Epstein, T. Canero, S. Christianson (Vancouver); J. Smith (Calgary); L. Morrison (Edmonton); M. Hader, D. Heiser (Saskatoon); B. Davis (Winnipeg); M. Perera (Toronto); P. Gaudet, C. Edgar (Kingston); D. Slavik, J. Haynes (Hamilton); R. Julien-Bimm, K. Stevenson (Ottawa); M. Lemieux, R. Arnaoutelis (Montreal - MNI); C. Masse (Montreal - Notre Dame); F. Gosselin (Quebec City); M. Morash, P. Weldon (Halifax) and G. Alcock (St. John's).

We would like to specially thank the following from the London, Ontario Coordinating Centre: Holly Armstrong, Dave Dyment, Keith Cousins and Marian Young.

We would like to specially thank the following from the Vancouver, British Columbia Coordinating Centre: Erin Black, Irene Yee, Nancy Greig, Sylvie Bourque, Randy Holmes, Laila Abulhusn, Kathy Eisen, Faye Pileberg, Chris Bajdik, C. Cheung and Drs. Lorne Kastrukoff, John Hooge and Virginia Devonshire for their ongoing assistance.

*The two coordinating MS clinics are listed first. Other clinics are listed geographically by province, west to east. 


\section{REFERENCES}

1. Ebers GC, Sadovnick AD, Risch NJ and the Canadian Collaborative Study Group. Familial aggregation in multiple sclerosis is genetic. Nature 1995; 377: 150-151.

2. Sadovnick AD, Ebers GC, Dyment D, et al. Evidence for the genetic basis of multiple sclerosis. Lancet 1996; 347: 1728-1730.

3. Ebers GC, Kukay K, Bulman DE, et al. A full genome search in multiple sclerosis. Nature Genet 1996; 13: 472-476.

4. Ebers GC, Bulman DE, Sadovnick AD, et al. A population-based twin study in multiple sclerosis. N Engl J Med 1986; 315: 16381642.

5. Sadovnick AD, Armstrong H, Rice GPA, et al. A population-based study of multiple sclerosis in twins: update. Ann Neurol 1993; 33: 281-285.

6. Mumford CJ, Wood NW, Kellar-Wood $\mathrm{H}$, et al. The British Isles survey of multiple sclerosis in twins. Neurology 1994; 44: 11-15.

7. Sadovnick AD, Baird PA, Ward RH. Multiple sclerosis: Updated risks for relatives. Am J Med Genet 1988; 29: 533-541.

8. Carton H, Vlietinck R, Debruyne J, et al. Risks of multiple sclerosis in relatives of patients in Flanders, Belgium. J Neurol Neurosurg Psychiatry 1997; 62: 329-333.
9. Robertson NM, Fraser M, Deans J, et al. Age-adjusted recurrence risks for relatives of patients with multiple sclerosis. Brain 1996; 119: 449-455.

10. Sawcer S, Jones HB, Feakes R, et al. A genome screen in multiple sclerosis reveals susceptibility on chromosome $6 \mathrm{p} 21$ and $17 \mathrm{q} 22$. Nature Genet 1996; 13: 464-468.

11. The Multiple Sclerosis Genetics Group. Complete genomic screen for multiple sclerosis underscores a role for the major histocompatibility complex. Nature Genet 1996; 13: 469-471.

12. Robertson NP, O'Riordan J, Clayton D, et al. Conjugal multiple sclerosis. J Neurol 1995; 242: S8.

13. Sibley WA, Bamford CR, Clark K. Triggering factors in multiple sclerosis. In: Poser CM, Paty DW, Scheinberg L, et al., eds. The Diagnosis of Multiple Sclerosis. New York: Thieme-Stratton Inc., 1984; chap 2, 15.

14. Ebers GC, Sadovnick AD. Epidemiology. In: Paty DE, Ebers GC eds. Multiple Sclerosis. Philadephia: FA Davis Company, 1997; chap 2, 16-17.

15. Poser CM, Paty DW, Scheinberg L, et al. New diagnostic criteria for multiple sclerosis: Guidelines for research protocols. Ann Neurol 1983; 13: 227-231.

16. Kurtzke JF. Rating neurological impairment in multiple sclerosis: an expanded disability status scale (EDSS). Neurology 1983; 33: 1444-1452. 